Letter to the Editor: Comparison of two coarse fractionated radiation protocols for the management of canine pituitary macrotumor: an observational study of 24 dogs, Marcinowska et al., doi: 10.1111/vru.12270

Rohrer Bley, Carla

DOI: https://doi.org/10.1111/vru.12314

Posted at the Zurich Open Repository and Archive, University of Zurich ZORA URL: https://doi.org/10.5167/uzh-129695

Journal Article

Accepted Version

Originally published at:

Rohrer Bley, Carla (2016). Letter to the Editor: Comparison of two coarse fractionated radiation protocols for the management of canine pituitary macrotumor: an observational study of 24 dogs, Marcinowska et al., doi: 10.1111/vru.12270. Veterinary Radiology Ultrasound, 57(2):107-109.

DOI: https://doi.org/10.1111/vru.12314 


\section{Letter to the Editor: Comparison of Two Coarse Fractionated Radiation Protocols for the Management of Canine Pituitary Macrotumor: an Observational Study of 24 Dogs, Marcinowska et al., doi: 10.1111/vru.12270}

\section{Dear Editor,}

With interest I have read the article by Marcinowska and colleagues titled "Comparison of two coarse fractionated radiation protocols for the management of canine pituitary macrotumor: an observational study of 24 dogs". In this publication, the authors report the outcome of patients with pituitary macrotumors treated with a protocol consisting of either 10x3.8 Gy or $1 \mathrm{x} 5 \mathrm{~Gy}+4 \mathrm{x} 8.25 \mathrm{~Gy}$. I have to raise a major concern regarding the latter of the radiation protocols used: under the described technical conditions, the dose per fraction is too high and the justification with the given BED calculation does not adequately impress this risk upon the reader. While I understand that for groups without daily access to treatment machines and/or the need of manual calculation of the treatment fields, a reduction in fraction number and conformity have to be made, the general basic principles of dose and fractionation should still be considered.

For the protocol of $1 \times 5 \mathrm{~Gy}+4 \mathrm{x} 8.25 \mathrm{~Gy}$ used herein, my criticism is as follows: the authors state a BED of $138.5 \mathrm{~Gy}$, and they compare this number to other published protocols. ${ }^{1-3} \mathrm{~A}$ basic explanation of the "BED" is attempted. While the authors set the BED of $138.5 \mathrm{~Gy}$ in relation to applied protocol fractionation schedules in older publications, no quantification of the consequences (e.g. endpoint; "isoeffect") in terms of normal tissue complication probability (NTCP) of such a protocol is given, rendering the BED to a purely quantitative number. This 
is one of the known disadvantages of the BED-concept as a measure of treatment intensity: the derived number is numerically much greater than any "real" prescribeable radiation dose and therefore is not easy to relate to everyday clinical practice.

By using the simpler concept (mathematically and biologically equivalent) of $\mathrm{EQD}_{2}{ }^{4}$, treatment schedules that have differing doses per fraction can be compared with much more relatable derived doses. These doses can further readily be compared to toxicity data published for tissues and endpoints in human medicine. Hence a fast, albeit rough comparison for organ tolerance can be made for a protocol, if an extensive NTCP-calculation cannot be made. Such a comparison needs the generous assumption that canine neuronal tissue is equally sensitive to ionizing radiation as human neuronal tissue, which is a limitation of this approach. However the impact of the comparison of $\mathrm{EQD}_{2}$ values communicates more effectively the potential for toxicity than comparison of BED values.

With the manual calculation of the fields and the portal sizes used in the publication of Marcinowska et al., volumes of about $20-40 \mathrm{~cm}^{3}$ can be derived as "treated volume" (e.g. the volume enclosed by an isodose surface, selected and specified as being appropriate to achieve the purpose of treatment $)^{5,6}$. Regular canine brain volumes range in the magnitude of $82+/-$ $19 \mathrm{~cm}^{3},{ }^{7}$ in consequence, this dose was applied to at least an approximate $1 / 3$ to $1 / 2$ of the total brain volume in most of the cases.

Tabulated below the BED and $\mathrm{EQD}_{2}$ of the protocols mentioned in the publication

\begin{tabular}{lcccccc}
\hline & $10 \times 3.8 \mathrm{~Gy}$ & $1 \mathrm{x} 5 \mathrm{~Gy}+4 \mathrm{x} 8.25 \mathrm{~Gy}$ & $5+7+8+9+9 \mathrm{~Gy}^{1}$ & $12 \times 4 \mathrm{~Gy}^{3}$ & $16 \mathrm{x} 3 \mathrm{~Gy}^{2}$ & $20 \mathrm{x} 2.5 \mathrm{~Gy}^{8}$ \\
\hline Total dose & $38 \mathrm{~Gy}$ & $38 \mathrm{~Gy}$ & $38 \mathrm{~Gy}$ & $48 \mathrm{~Gy}$ & $48 \mathrm{~Gy}$ & $50 \mathrm{~Gy}$ \\
\hline BED $_{(\alpha / \beta=3)}$ & 86.1 & 138.5 & 137.9 & 110.4 & 96 & 91.6 \\
\hline $\mathrm{EQD}_{2(\alpha / \beta=3)}$ & 51.7 & 82.3 & 82.2 & 67.2 & 57.6 & 55 \\
\hline
\end{tabular}


If now we compare these numbers $\left(\mathrm{EQD}_{2}\right)$ to the tabulated numbers of the old, but still frequently used reference for $\mathrm{TD}_{5 / 5}$ and $\mathrm{TD}_{50 / 5},{ }^{9}$

\begin{tabular}{|c|c|c|c|c|c|c|c|}
\hline \multirow[b]{2}{*}{ Volume } & \multicolumn{3}{|c|}{$\mathrm{TD}_{5 / 5} *$ Volume } & \multicolumn{3}{|c|}{$\mathrm{TD}_{50 / 5}$ Volume } & \multirow[b]{2}{*}{ Endpoint } \\
\hline & $1 / 3$ & $2 / 3$ & $3 / 3$ & $1 / 3$ & $2 / 3$ & $3 / 3$ & \\
\hline Brain & 60 & 50 & 45 & 75 & 65 & 60 & Necrosis \\
\hline Chiasma & No pa & olume & 50 & No pr & olume & 65 & Blindness \\
\hline
\end{tabular}

these numbers lead to the - admittedly very rough - conclusion, that the risk of inducing brain necrosis with the $1 \times 5 \mathrm{~Gy}+4 \mathrm{x} 8.25 \mathrm{~Gy}$ protocol $\left(\mathrm{EQD}_{2}\right.$ of 82.3$)$ could be substantial.

As suggested in the QUANTEC Report, Emami's estimate for fractionated partial brain irradiation $\left(\mathrm{TD}_{5 / 5}\right.$ for $1 / 3$ of the brain volume, $60 \mathrm{~Gy}$ ) may be overly conservative. ${ }^{10-12}$ More recent data suggest a $\mathrm{TD}_{5 / 5}$ (partial) of $72 \mathrm{~Gy}$, in $2 \mathrm{~Gy}$ fractions, which confers to a BED of about $120 \mathrm{~Gy} .{ }^{10}$ This number, however, exceeds the tolerance for the optic chiasm ${ }^{11}$ and the brain stem, ${ }^{12}$ which are anatomically flanking the area of a pituitary tumor. Furthermore, given the very crude method of target delineation, treatment planning and delivery of radiotherapy (single-slice 2D MR planning and no position verification) in the study of Marcinowska et al., it is probably justified to assume a more conservative tolerance dose. In addition, as the brain is especially sensitive to fraction sizes $>2 \mathrm{~Gy}$, severely hypofractionated regimes will probably be underestimated by $\mathrm{EQD}_{2}$ or $\mathrm{BED}$ calculations regarding possible late complications.

While the authors mention the possibility of radiation injury caused by this protocol, not much emphasis is given to this "possibility" as the observed complications occurred very 
early rather than after the expected time delay of several months to years which is typical for late toxicity. Unfortunately, no evaluation for late toxicity is provided for this in this study, rendering the deaths of these patients futile. MRI and/or postmortem histopathology should have been included in at least a part of these patients.

For this study, it must be assumed that the $1 \times 5 \mathrm{~Gy}+4 \mathrm{x} 8.25 \mathrm{~Gy}$ protocol carries a high risk for complications and that the incredibly early occurrence of clinical signs implicates a severe breach in radiobiological considerations of this protocol given the non-conformal, nonverified dose delivery technique. In my personal view, using a protocol with a dose equivalent that exeeds advisable toxicity ranges should not be attempted without appropriate use of conformal techniques at the cost of sick patients. Canine pituitary macroadenoma is a disease that undoubtedly carries a very favorable prognosis if treated within the calculable risk. ${ }^{2,7}$ For colleagues using radiation therapy, delivering the prescribed dose to a defined target, while maintaining dose within tolerance to normal tissues should remain the overarching principle of clinical practice.

Prof. Dr. med. vet. Carla Rohrer Bley

DACVR (Radiation Oncology), DipECVDI (add Rad Oncol)

MAE (Applied Ethics)

Head of Oncology

Division of Radiation Oncology, Vetsuisse Faculty, University of Zurich

Winterthurerstrasse 258c, CH-8057 Zürich, Switzerland 


\section{References}

1. Brearley MJ, Jeffery ND, Phillips SM, Dennis R. Hypofractionated radiation therapy of brain masses in dogs: a retrospective analysis of survival of 83 cases (1991-1996). J Vet Intern Med. 1999;13: 408-412.

2. Kent MS, Bommarito D, Feldman E, Theon AP. Survival, neurologic response, and prognostic factors in dogs with pituitary masses treated with radiation therapy and untreated dogs. J Vet Intern Med. 2007;21: 1027-1033.

3. Theon AP, Feldman EC. Megavoltage irradiation of pituitary macrotumors in dogs with neurologic signs. J Am Vet Med Assoc. 1998;213: 225-231.

4. Joiner MC, Bentzen SM. Fractionation: the linear-quadratic approach. In: Joiner MC, Van der Kogel A(eds): Basic Clinical Radiobiology. Great Britain: Edward Arnold, 2009;102-119.

5. International Commission of Radiation Units and Measurements. Prescribing, recording, and reporting photon beam therapy, ICRU Report 50. Bethesta, MD: ICRU, 1993. 6. International Commission of Radiation Units and Measurements. Prescribing, recording, and reporting photon beam therapy (Supplement to ICRU Report 50), ICRU Report 62. Bethesda, MD: ICRU, 1999.

7. Rohrer Bley C, Sumova A, Roos M, Kaser-Hotz B. Irradiation of brain tumors in dogs with neurologic disease. J Vet Intern Med. 2005;19: 849-854.

8. Keyerleber MA, McEntee MC, Farrelly J, Thompson MS, Scrivani PV, Dewey CW. Three-dimensional conformal radiation therapy alone or in combination with surgery for treatment of canine intracranial meningiomas. Vet Comp Oncol. 2013.

9. Emami B, Lyman J, Brown A, Coia L, Goitein M, Munzenrider JE, et al. Tolerance of normal tissue to therapeutic irradiation. Int J Radiat Oncol Biol Phys. 1991;21: 109-122. 
10. Lawrence YR, Li XA, el Naqa I, Hahn CA, Marks LB, Merchant TE, et al. Radiation dose-volume effects in the brain. Int J Radiat Oncol Biol Phys. 2010;76: S20-27.

11. Mayo C, Martel MK, Marks LB, Flickinger J, Nam J, Kirkpatrick J. Radiation dosevolume effects of optic nerves and chiasm. Int J Radiat Oncol Biol Phys. 2010;76: S28-35.

12. Mayo C, Yorke E, Merchant TE. Radiation associated brainstem injury. Int J Radiat Oncol Biol Phys. 2010;76: S36-41. 